\title{
Glycerol Esterification with Acetic Acid by Reactive Distillation Using Hexane as an Entrainer
}

\author{
Tatiane F. C. Souza, Newton L. Ferreira, Maristhela Marin, and Roberto Guardani
}

\begin{abstract}
A process design study was carried out, aimed at the design of a triacetin production process from glycerol, as a way to increase the feasibility of biodiesel production. Glycerol esterification with acetic acid involves three consecutive reversible acetylation reactions and in each step, water is produced, resulting in limited conversion and low selectivity [1]. One way to increase the triacetin selectivity is to continuously remove water from the reaction medium, in order to shift the equilibrium. The proposed process is based on the reaction system described by Galan et al. [2], consisting of the esterification of glycerol using excess acetic acid as catalyst. In the first step of the present study an evaluation of the kinetic parameters was carried out, based on published experimental data [2]. The reaction conditions were then evaluated in terms of glycerol conversion and selectivity for different reaction times and temperatures. Based on the results, the process was simulated in a reactive distillation column, and different configurations were studied by using the Aspen Plus ${ }^{\circledR}$ simulator. In the separation units the NRTL-HOC equilibrium model with binary interaction parameters proposed by Hung et al. [3] was adopted. Water removal from the top stream of the column was increased by feeding hexane as an entrainer in the reactive distillation column. Hexane is recovered in a separate unit and recycled to the process. The conceptual process specifications of an optimized industrial plant configuration were estimated for minimum specific energy consumption for production of $99.9 \%$ molar purity triacetin with complete glycerol conversion.
\end{abstract}

Index Terms-Glycerol esterification, triacetin, reactive distillation, simulation.

\section{INTRODUCTION}

Glycerol is a by-product from the esterification of vegetable oils and fats, and is generated at a ratio of ca. $0.1 \mathrm{~kg}$ per $\mathrm{kg}$ of biodiesel produced. In recent years, mainly due to biodiesel production, the available quantity of glycerol in the market is increasing significantly, and many processes that use glycerol are being studied and developed. Triacetin production through the esterification of glycerol with acetic acid is one of the possible routes to transform glycerol into valuable products, since triacetin has a market possibility as plasticizer and fuel additive. It has been reported that adding triacetin to the biodiesel can reduce CO emissions [4] and improve the biodiesel flowability under low temperatures. Glycerol esterification with acetic acid is composed of three consecutive equilibrium reactions, and is carried out in the

Manuscript received August 30, 2017; revised November 20, 2017.

Tatiane Fernandes, Caetano Souza, and Roberto Guardani are with Escola Politécnica da Universidade de São Paulo, Brazil (e-mail:tatianefcsouza@gmail.com,guardani@usp.br).

Newton Libanio Ferreira and Maristhela Marin are with Centro Universitário FEI, Brazil (e-mail: newton.libanio@uol.com.br, marimari@fei.edu.br). presence of an acidic catalyst. In each acetylation step water is produced.

$$
\begin{gathered}
\text { Glycerol + Acetic Acid } \leftrightarrow \text { Monoacetin + Water } \\
\text { Monoacetin }+ \text { Acetic Acid } \leftrightarrow \text { Diacetin }+ \text { Water } \\
\text { Diacetin }+ \text { Acetic Acid } \leftrightarrow \text { Triacetin }+ \text { Water }
\end{gathered}
$$

The presence of water limits glycerol conversion and triacetin selectivity, and this has motivated studies aimed at identifying favorable conditions for this reaction system, in terms of temperature, acetic acid/glycerol ratio, pressure and catalyst concentration. In order to reduce the impact of the water formation, Liao et al. studied the use of acetic anhydride instead of acetic acid, concluding that thermodynamically the triacetin production with this reagent is more favorable than with acetic acid [5]. However, acetic anhydride is relatively expensive, and its use can make the production process unfeasible in economic terms. Gonçalves et al. analyzed the catalysis performance of different catalysts, such as ion-exchange resins (Amberlyst-15, K-10, HZSM-5 and HUSY) and niobic acid. The study showed that the Amberlyst-15 resin was the one that presented the highest glycerol conversion (97\%) [6].

There are not many published studies on the kinetics of the reaction system. Mufrodi et al. studied the batch triacetin production catalyzed by sulfuric acid and obtained $77.84 \%$ triacetin selectivity [7]. The kinetic parameters, based on the Arrhenius model, were obtained for the six reactions (direct and reverse reactions of each acetylation step). Following this same model, Galan et al. studied the reaction kinetics for the esterification of glycerol with excess of acetic acid as catalyst, as a way to shift the equilibrium to the products [2].

Hung et al. designed a reactive distillation (RD) column to produce triacetin from glycerol, and in order to increase the water removal at the top of the RD column, isobutyl acetate and ethylene dichloride were used as entrainers. The effect of the entrainer was evaluated for both processes. The authors concluded that the process using entrainer can reduce the total annual production cost by more than $34 \%$ [3].

In the present study, the kinetic parameters obtained by Galan et al. [2] for reactions 1 to 3 were evaluated and then used in the simulation of an industrial process based on reactive distillation. However, in this case hexane was adopted as entrainer to promote water removal at the top of the column. In the separation units the NRTL-HOC equilibrium model with binary interaction parameters proposed by Hung et al. [3] was adopted. The process conditions that lead to high glycerol conversion and high purity triacetin were studied by means of simulations for 
different process configurations, in order to identify the optimal conditions in terms of minimum energy consumption. Finally, a triacetin production unit that uses glycerol produced in a typical biodiesel plant was proposed.

All simulations and parameter fitting studies were performed in Scilab and Aspen Plus simulation platforms.

\section{REACTION KINETICS}

Considering a batch pressurized reactor, Galan et al [2] carried out experimentally the reaction of glycerol with excess acetic acid (proportion 1:12, i.e., four times in excess) at two temperatures, namely $120^{\circ} \mathrm{C}$ and $160{ }^{\circ} \mathrm{C}$, under ca. 10 bar pressure, in order to prevent acetic acid evaporation during the reaction and thus decrease glycerol conversion. The three consecutive equilibrium reactions can be represented as six direct reactions, as follows:

$$
\begin{array}{ll}
\text { 1) } & \mathrm{G}+\mathrm{A} \stackrel{\mathrm{k} 1}{\rightarrow} \mathrm{M}+\mathrm{W} \\
\text { 2) } & \mathrm{M}+\mathrm{W} \stackrel{\mathrm{k} 2}{\rightarrow} \mathrm{G}+\mathrm{A} \\
\text { 3) } & \mathrm{M}+\mathrm{A} \stackrel{\mathrm{k} 3}{\rightarrow} \mathrm{D}+\mathrm{W} \\
\text { 4) } & \mathrm{D}+\mathrm{W} \stackrel{\mathrm{k} 4}{\rightarrow} \mathrm{M}+\mathrm{A} \\
\text { 5) } & \mathrm{D}+\mathrm{A} \stackrel{\mathrm{k} 5}{\rightarrow} \mathrm{T}+\mathrm{W} \\
\text { 6) } & \mathrm{T}+\mathrm{W} \stackrel{\mathrm{k} 6}{\rightarrow} \mathrm{D}+\mathrm{A}
\end{array}
$$

The symbols are G: glycerol; A: acetic acid; M: monoacetin; D: diacetin; T: triacetin and W: water.

The second order reaction rates are represented as:

$$
\begin{aligned}
& r_{1}^{\prime}=k_{1} \cdot C_{A} \cdot C_{G} \\
& r_{2}{ }_{2}=k_{2} \cdot C_{M} \cdot C_{W} \\
& r^{\prime}{ }_{3}=k_{3} \cdot C_{M} \cdot C_{A} \\
& r{ }_{4}=k_{4} \cdot C_{D} \cdot C_{W} \\
& r_{5}{ }_{5}=k_{5} \cdot C_{D} \cdot C_{A} \\
& r{ }_{6}=k_{6} \cdot C_{T} \cdot C_{W}
\end{aligned}
$$

\begin{tabular}{|c|c|c|}
\hline Temperature & $120^{\circ} \mathrm{C}$ & $160{ }^{\circ} \mathrm{C}$ \\
\hline$k_{1}$ & $2,38 \mathrm{E}-04$ & $2,56 \mathrm{E}-04$ \\
\hline$k_{2}$ & 1,20E-01 & 7,62E-02 \\
\hline$k_{3}$ & $6,30 \mathrm{E}-04$ & $5,30 \mathrm{E}-04$ \\
\hline$k_{4}$ & 3,14E-03 & 8,78E-03 \\
\hline$k_{5}$ & 1,09E-04 & 1,96E-04 \\
\hline$k_{6}$ & 2,59E-03 & 4,76E-03 \\
\hline
\end{tabular}

With the kinetic constants for reaction $i$ expressed according to the Arrhenius model:

$$
k_{i}=A_{i} \cdot e^{\frac{-E_{i}}{R \cdot T}}
$$

where $A_{i}$ is the pre-exponential factor and $E_{i}$ is the activation energy for each reaction.

The experimental values of these constants were fitted from the experimental data at each temperature, and are shown in Table I.

TABLE I: AdJUSTED KinETIC PARAMETERS [L/MOL.S] OBT AINED BY GALAN ET AL. [2]

For two temperatures, the system of equations is determined, and thus the two parameters are directly obtained for each rate constant, as shown in Table II.
These constants were evaluated by simulating a batch reactor under similar conditions to those adopted by Galan et al. [2]. i.e., by feeding $22 \mathrm{~mL}$ glycerol and $208 \mathrm{~mL}$ acetic acid to the reactor, and 8 hours of reaction time. The system equilibrium was achieved after around 15 minutes of reaction at $120{ }^{\circ} \mathrm{C}$, and around 10 minutes at $160{ }^{\circ} \mathrm{C}$. The concentration of the species during the reaction time is shown in Figs. 1 and 2, for $120^{\circ} \mathrm{C}$ and $160{ }^{\circ} \mathrm{C}$, respectively.

TABLE II: ARRHENIUS PARAMETERS FOR THE REACTION SYSTEM

\begin{tabular}{lll}
\hline & $A_{i}(\mathrm{~L} / \mathrm{mol} . \mathrm{s})$ & $E_{i}(\mathrm{cal} / \mathrm{mol})$ \\
\hline Reaction 1 & $5,24 \mathrm{E}-04$ & $6,17 \mathrm{E}+02$ \\
Reaction 2 & $8,56 \mathrm{E}-04$ & $-3,86 \mathrm{E}+03$ \\
Reaction 3 & $9,69 \mathrm{E}-05$ & $-1,46 \mathrm{E}+03$ \\
Reaction 4 & $2,16 \mathrm{E}+02$ & $8,70 \mathrm{E}+03$ \\
Reaction 5 & $6,26 \mathrm{E}-02$ & $4,96 \mathrm{E}+03$ \\
Reaction 6 & 1,86 & $5,14 \mathrm{E}+03$ \\
\hline \hline
\end{tabular}

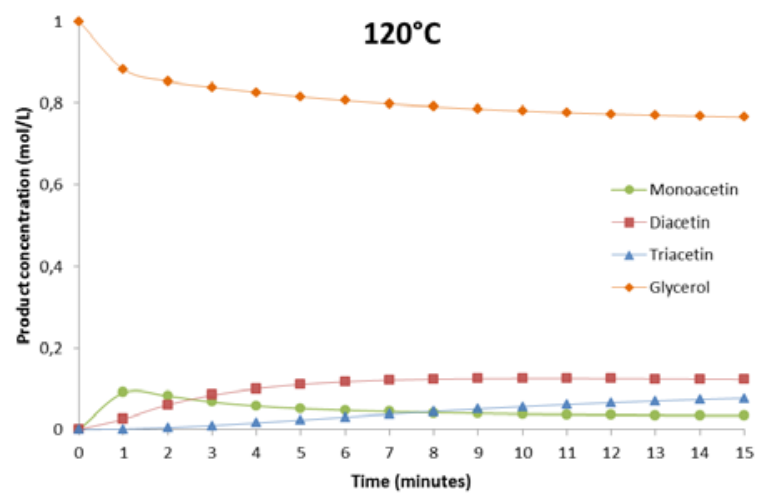

Fig. 1. Species concentration over reaction time for the simulated reactor at $120{ }^{\circ} \mathrm{C}$, considering the Arrhenius parameters obtained by Galan et al.[2].

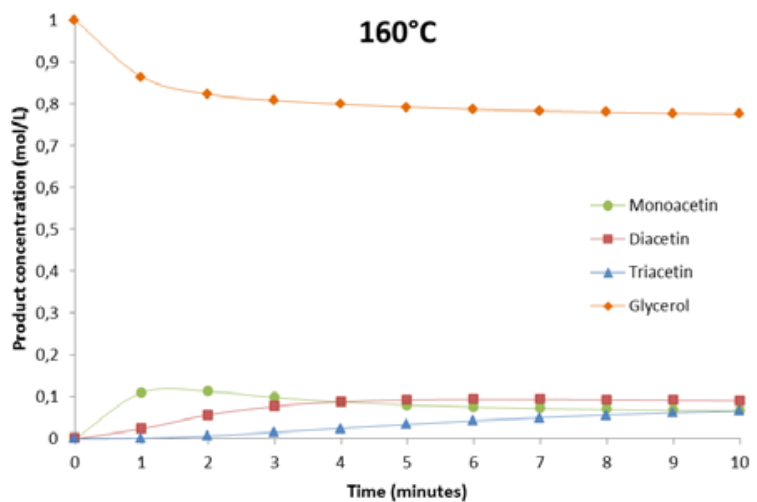

Fig. 2. Species concentration over reaction time for the simulated reactor at $160{ }^{\circ} \mathrm{C}$, considering the Arrhenius parameters obtained by Galan et al.[2].

As shown in the plots, the maximum glycerol conversion was ca. $25 \%$ for both temperatures, and, although the product distribution is different, conversion to triacetin remained low in both cases. This is a clear evidence of the effect of the presence of water in the system, indicating the need for water removal, which can be accomplished by reactive distillation, as suggested by Galan et al [2].

\section{PROCESS DESIGN}

\section{A. Methods}

The design of the triacetin production process was based on the following criteria:

1) the adopted triacetin plant capacity is based on a 
typical biodiesel production facility in Brazil, with an average installed capacity to produce ca. $400 \mathrm{~m}^{3}$ of biodiesel per day [8], and around $1500 \mathrm{~kg} / \mathrm{h}$ of glycerol (17 kmol/h approximately); this is the adopted glycerol flowrate in the feed stream;

2) The product stream contains minimum $99 \%$ of triacetin (molar base);

3) Among the components involved in the process, monoacetin and diacetin are not present in the properties databank of the simulator. Thus, the parameters for the equilibrium correlations were estimated by the simulator from the molecular structures of these two species, which were fed to the software. Due to their molecular structures, the most favorable isomers to form during the reactions are 1-monoacetin and 1,3-diacetin [9], and thus these isomers were adopted to represent the monoacetin and diacetin in the simulations;

4) the NRTL-HOC thermodynamic model was adopted, because it can well represent aqueous electrolyte systems, and in addition, the Hayden-O'Connell model provides an adequate representation of the vapor phase behavior, since during the reaction gaseous species are generated, mainly due to acetic acid evaporation; the binary interaction parameters were the same ones adopted by Hung et al. [3];

5) The reaction rates were modeled according to the previously described kinetic model, with the kinetic parameters shown in Table II. As in the study by Galan et al. [2], four times molar excess of acetic acid was adopted, so that $1 \mathrm{~mol}$ of glycerol to $12 \mathrm{~mol}$ of acetic acid was fed to the reactive distillation column.

6) The two reagent streams are assumed to contain $5 \%$ of water as impurity (mol base), as adopted by Hung et al. [3]. The specifications of these streams are listed in Table III.

7) Hexane was used as a water entrainer in the column, due to its low solubility in water, which can improve its separation and recovery, and also because hexane forms an azeotrope with water at a moderate temperature $\left(49.2^{\circ} \mathrm{C}\right)$, which can facilitate water entrainment.

The next section describes the design of the process.

\section{B. Proposed Triacetin Production Process}

The processing units and streams of the proposed triacetin production process are represented by the flowsheet shown in Fig. 3. The process consists of recycle circuits for hexane (entrainer) and for the excess acetic acid, as well as for the bottom stream from the reactive distillation column, as a way to convert all the glycerol and reaction intermediates, and to produce a product stream with more than $99 \%$ triacetin. The main unit operations are:

C1 - Reactive distillation column, in which the reactions take place, and water is removed by hexane in the top stream.

C2 - Acetic acid removal column, in which the excess acetic acid is removed in the top stream, and glycerol, monoacetin and diacetin are recovered in the bottom stream. This bottom stream is mixed with the bottom stream from column C3 and returned to the reactive column. Triacetin is removed in stream 7 , as an intermediate exit stream from column C2, specified according to the temperature profile along the column.

C3 - Triacetin purification column, in which the remaining glycerol, diacetin and monoacetin are removed in the bottom stream, which is returned to the reactive distillation column. The product stream 10 from the top of column C3 contains more than $99 \%$ molar fraction of triacetin.

$\mathrm{H} 1$ - Condenser of the reactive distillation column: The condenser of this column cannot be directly connected to the reactive column, since it is necessary to separate the hexane from the top stream, which it takes place at the decanter.

D1 - Decanter: The condensed top stream from the reactive distillation column is decanted to separate hexane from the solution of acetic acid in water.

M1 - Hexane mixer: The hexane stream recovered at the decanter is mixed with the hexane make-up stream forming the feed stream to the reactive distillation column.

M2 - Heavy components mixer: The bottom streams from columns C2 and C3, consisting mainly of glycerol, diacetin and monoacetin, are mixed and fed to the reactive distillation column.

M3 - Mixer of recovered acetic acid: The top stream from column C2 and the bottom stream from the decanter D1, which are rich in acetic acid, are mixed and sent to an acetic acid recovery unit, outside the limits of the triacetin production unit.

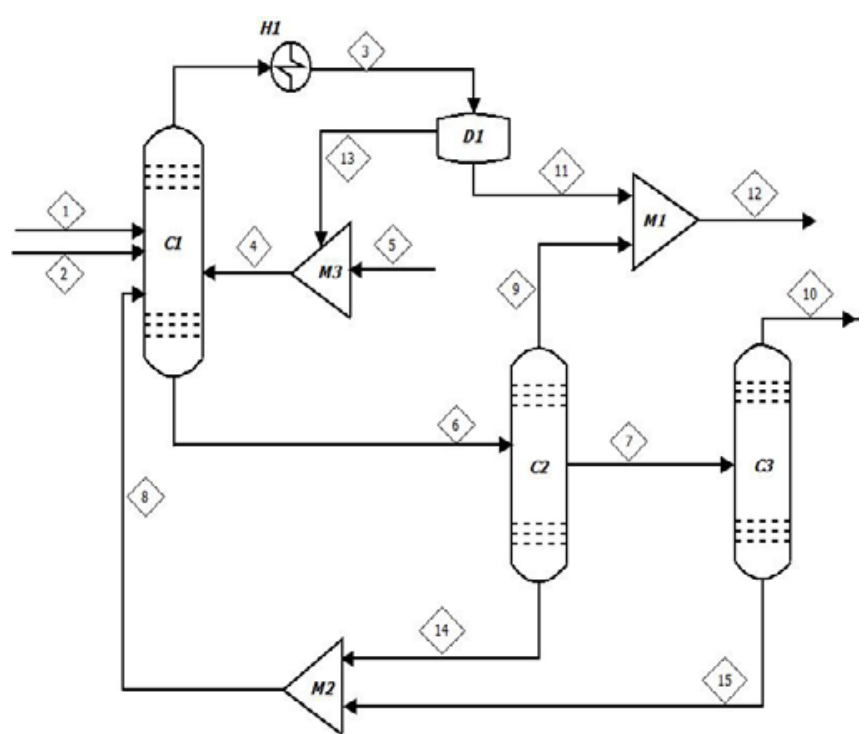

Fig. 3. Proposed flowsheet for the triacetin production process with hexane as an entrainer.

TABLE III: PROCESS FEED STREAMS

\begin{tabular}{clll}
\multicolumn{4}{c}{ TABLE III: PROCESS FEED STREAMS } \\
\hline \hline & & 1 & 2 \\
\hline Mole Flow & Acetic acid & 0 & 193,8 \\
$(\mathrm{kmol} / \mathrm{h})$ & Glycerol & 16,15 & 0 \\
& Water & 0,85 & 10,2 \\
Total Flow $(\mathrm{kmol} / \mathrm{h})$ & 17 & 204 \\
Total Flow $(\mathrm{kg} / \mathrm{hr})$ & 1502,6 & 11821,9 \\
Temperature $\left({ }^{\circ} \mathrm{C}\right)$ & 80 & 80 \\
Pressure $($ bar $)$ & 1 & 1 \\
State & Liquid & Liquid \\
\hline \hline
\end{tabular}

The streams numbered " 1 " and " 2 " in Fig. 3 are the feed of glycerol and acetic acid respectively. Stream " 5 " is the hexane make-up required to replace hexane losses in the hexane cycle. Stream " 8 " is the bottom recovery from 
columns C2 and C3, and is mainly composed of heavy components (glycerol, diacetin and monoacetin). This stream is returned to the reactive distillation column (C1) to continue the reaction to triacetin.

The operation conditions and main characteristics of the streams are discussed in the next section.

\section{Results and Discussion}

The reactive distillation column $\mathrm{C} 1$ is divided in two sections: reaction zone and separation zone. The reaction zone is composed of the bottom part of the column and is where the reaction takes place. The separation zone is in the top part of the column, where water is removed, with hexane as entrainer. The configuration of column C1 was designed in order to maximize the water removal at the top and glycerol conversion. The pressure in the column affects the gas-liquid equilibrium composition and temperature, and thus affects the maximum attainable glycerol conversion and triacetin selectivity at the bottom part, as shown in Fig. 4. Based on this behavior, the pressure in column $\mathrm{C} 1$ was set to 1 bar. Under this pressure water forms an azoetrope with hexane at $49.02{ }^{\circ} \mathrm{C}$, with molar composition of 0.33 water and 0.67 hexane. Although the water content is low at the azeotropic composition when compared to other common entrainers, such as isobutyl acetate and ethylene dichloride, hexane has the advantage of lower cost, and the water removal rate from the column is adequate to reach the conversion requirements.

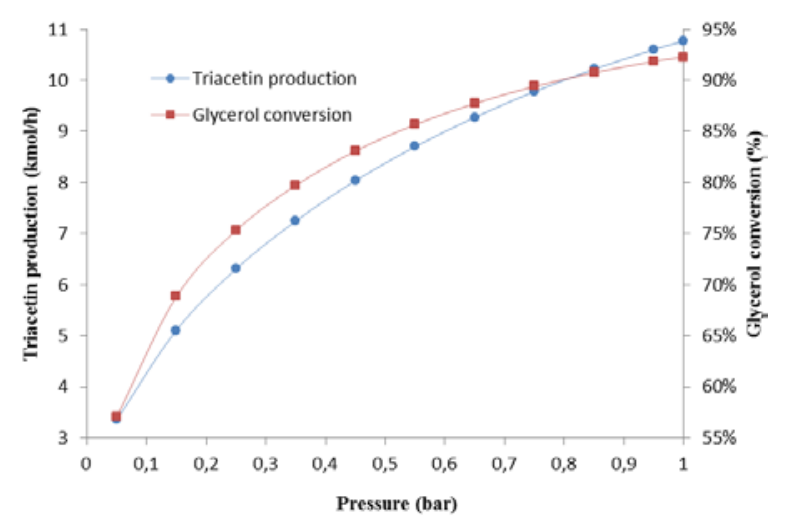

Fig. 4. Effect of $\mathrm{C} 1$ pressure on the triacetin production and glycerol conversion at the column.

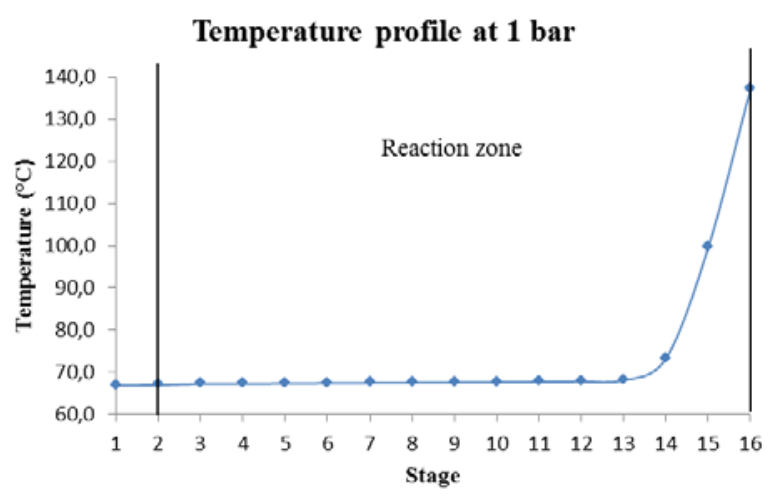

Fig. 5. Temperature profile of the reactive distillation column (C1).

A study was carried out in order to select a favorable reboiler duty, by varying the mass flow ratio of the bottom to feed streams. This resulted in a column with 16 stages, in which the $16^{\text {th }}$ stage consists of the reboiler. The condenser is a separate unit, since it is necessary to separate hexane from the water-acetic acid solution in a decanter prior to its return to the column. The feed streams (streams 1 and 2) are fed at stage 2 , and the hexane reflux (stream 4 ) is fed at stage 1 . The reaction zone is distributed over stages 2 to 16 , and the recover process stream (stream 8 ) is fed at stage 2, the same stage as the feed streams.

Fig. 5 shows the temperature profile in column C1. There is an increase of the temperature in the reaction zone, mainly at stage 16 , where the residence time is larger. This increase of the temperature is due to the exothermic reaction of glycerol to acetic acid [10], which favors the formation of the hexane-water stream.

The top stream is condensed and decanted. The outlet temperature of the condenser and at the decanter were studied in order to maximize the separation of water from hexane. Lower temperatures favor the water removal (Fig. 6). Based on these results, the temperature of the outlet stream from the condenser (stream 3) was set to $5{ }^{\circ} \mathrm{C}$ at 1 bar. The heat removal from the condenser is estimated at ca. 18.5 MW.

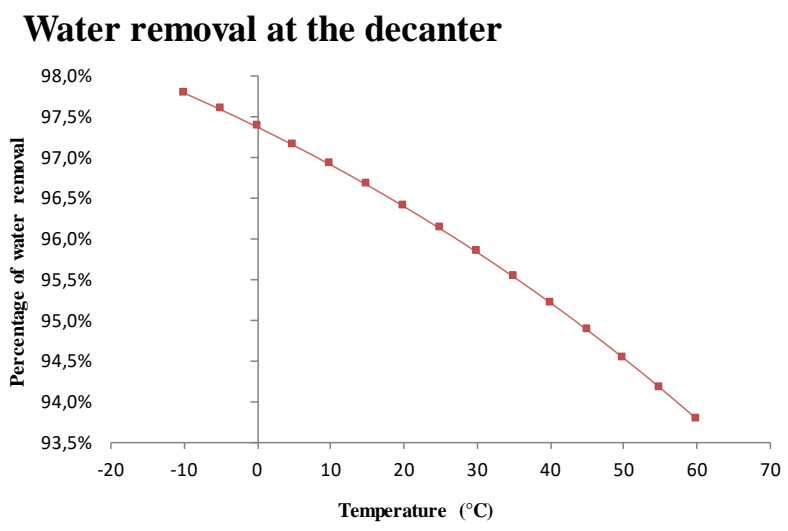

Fig. 6. Effect of temperature on the percentage of water removal at the decanter (1 bar).

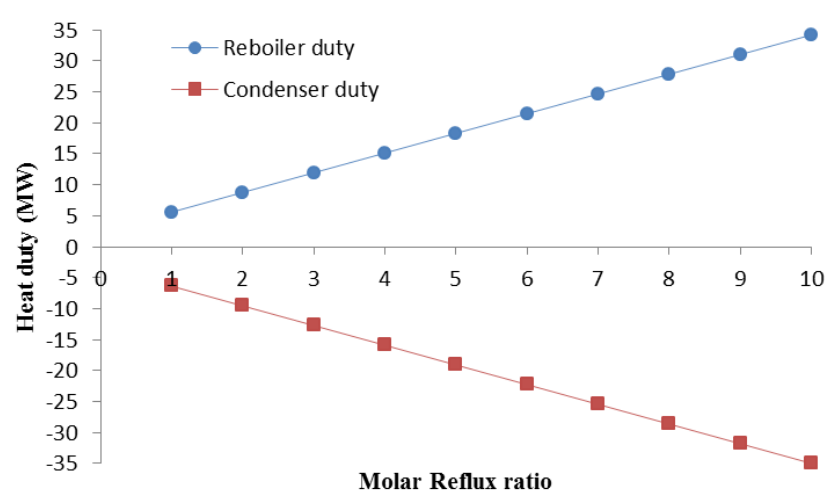

Fig. 7. Effect of molar reflux ratio on the condenser and reboiler heat duties in column $\mathrm{C} 2$.

Stream 3, which contains mainly hexane, is decanted, mixed to the hexane make-up stream and fed to column C1. The flowrate of the make-up stream is relatively small (20.5 $\mathrm{kg} / \mathrm{h}$ ), and replaces hexane losses in the decanter and at the bottom stream from C1.

The column C1 reboiler operates at $137.2^{\circ} \mathrm{C}$ and its heat duty is ca. $18 \mathrm{MW}$. The bottom stream is fed in the second column (C2) to remove the excess acetic acid.

In column C2 the excess acetic acid is removed. This excess in the process feed stream is necessary, since, as described previously, acetic acid is a reagent and a catalyst, too. Triacetin is not the heaviest component of the system, so it has to be withdrawn at an intermediate stage of the column, 
and glycerol, monoacetin and diacetin, heavier components, are recovered at the bottom of column C2. This column was designed in order to remove all the acetic acid excess at the top. The favorable conditions were estimated by simulating the column behavior for different distillate flowrates and reflux ratios, and intermediate product flowrates, which are directly related to the condenser and reboiler heat duties (Fig. 7). Lower pressures favor the acetic acid removal, since it reduces the amount of acetic acid in the product and the triacetin loss at the top (Fig. 8).

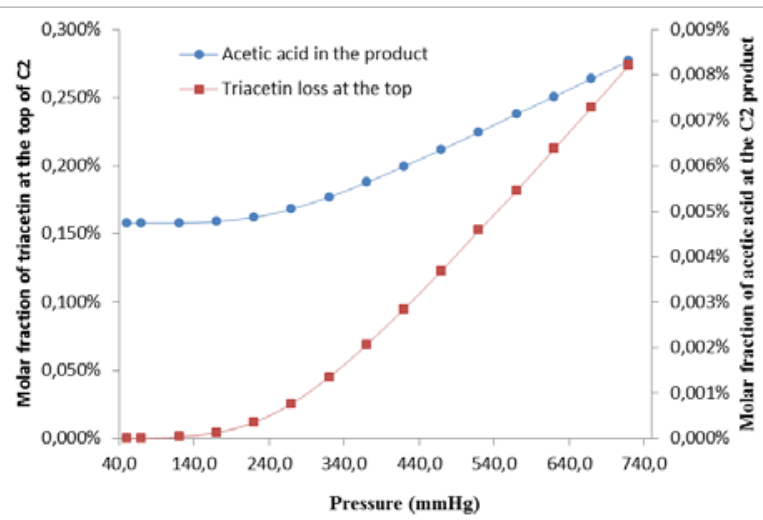

Fig. 8. Effect of pressure on the molar fraction of triacetin at the $\mathrm{C} 2$ top stream and molar fraction of acetic acid at the product stream from C2.

The optimal design, represented by minimum energy consumption and high product purity, resulted in a column with 15 stages, where the first stage is the condenser and the $15^{\text {th }}$ stage is the reboiler. The column pressure was set to 50 $\mathrm{mmHg}$ (0.07 bar) in order to favor the triacetin purity (Fig. 8). The feed enters at stage 3 , and the intermediate product is withdrawn at stage 5 (Fig. 9).

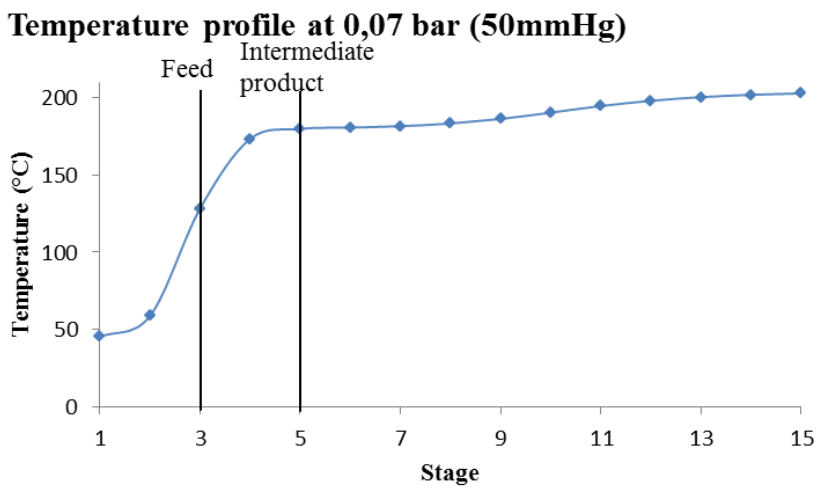

Fig. 9. Temperature profile of the acetic acid removal column (C2).

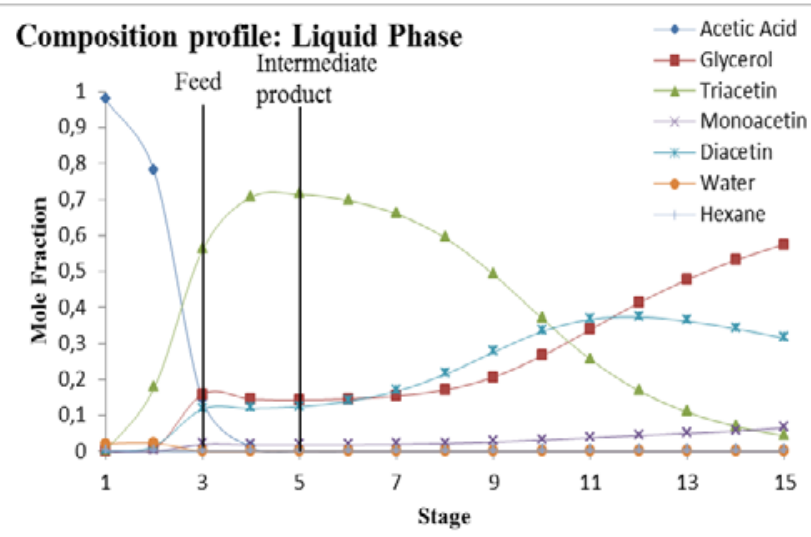

Fig. 10. Molar liquid composition profile at column C2.
The molar compositions of the vapor and liquid phases in the acetic acid removal column C2 are shown in Figs. 10 and 11). The triacetin mole fraction profile was the adopted criteria to specify the stage in which the intermediate stream 7 is placed. This stream (stream 7 in Fig. 3) has $72 \%$ molar fraction of triacetin, and the remaining components are mainly diacetin and glycerol. This stream is fed to column C3, where the remaining heavier compounds are removed in order to attend the required product specifications. The top stream from column $\mathrm{C} 2$ is rich in acetic acid, and is sent to the recovery unit. The bottom stream is rich in diacetin and glycerol. This stream is mixed with the bottom stream from column C3 and returned to the reactive distillation column (C1).

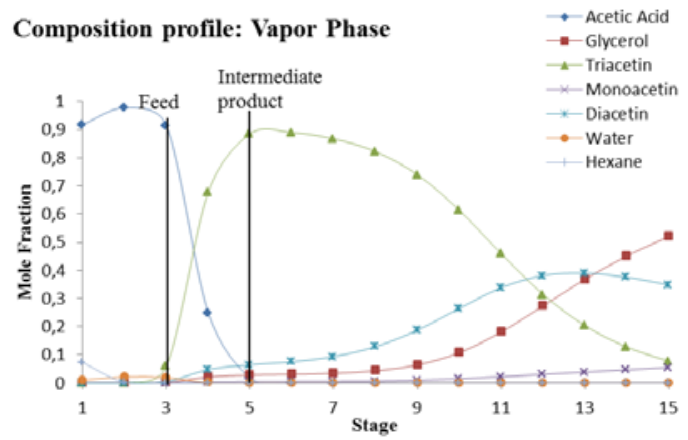

Fig. 11. Molar vapor composition profile at column C2.

The optimal reflux ratio in column C2 is 1 , the condenser removal duty is $1547.5 \mathrm{~kW}$, and the reboiler duty is $1430 \mathrm{~kW}$.

The triacetin purification column (C3) was designed in a way to guarantee that the top stream attends the triacetin purity level (more than $99 \%$ molar). The column pressure, distillate flowrate and reflux ratio, which are directly related to condenser and reboiler heat duties, were optimized in order to attend the required specifications (Fig. 12).

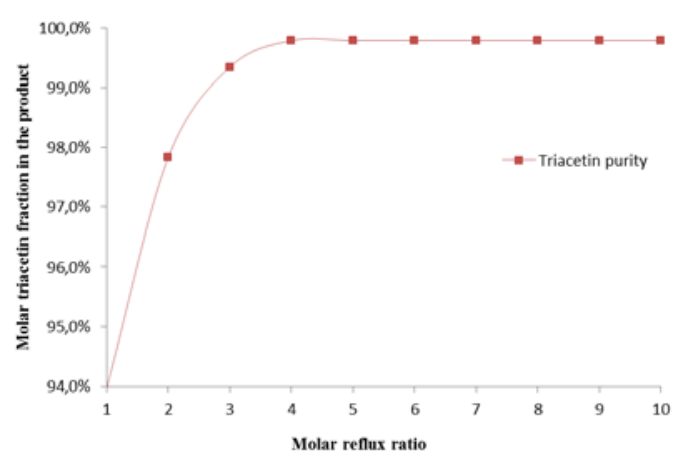

Fig. 12. Effect of the molar reflux ratio on the molar triacetin fraction in the product (purity).

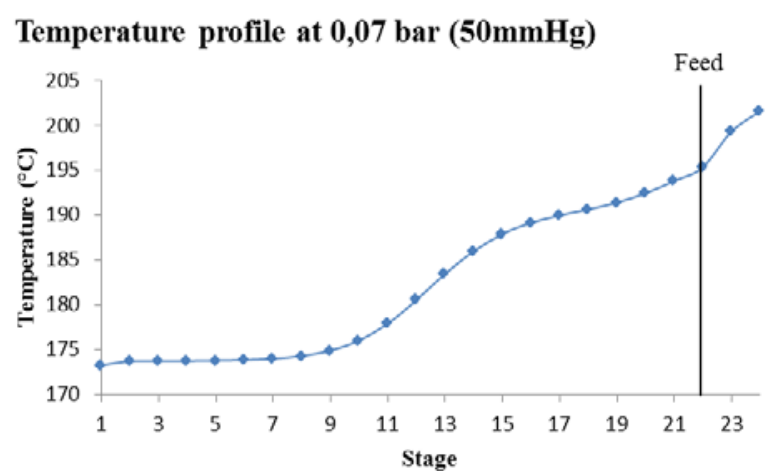

Fig. 13. Temperature profile of triacetin purification column (C3). 


\section{Composition profile: Liquid Phase}

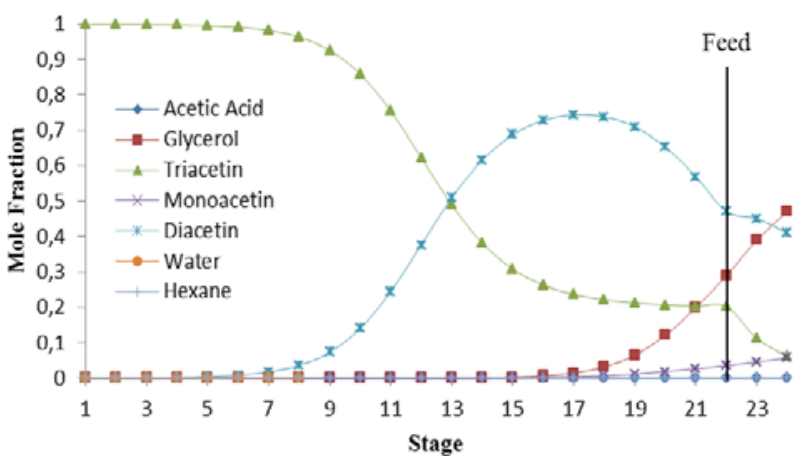

Fig. 14. Molar liquid composition profile at column C3.

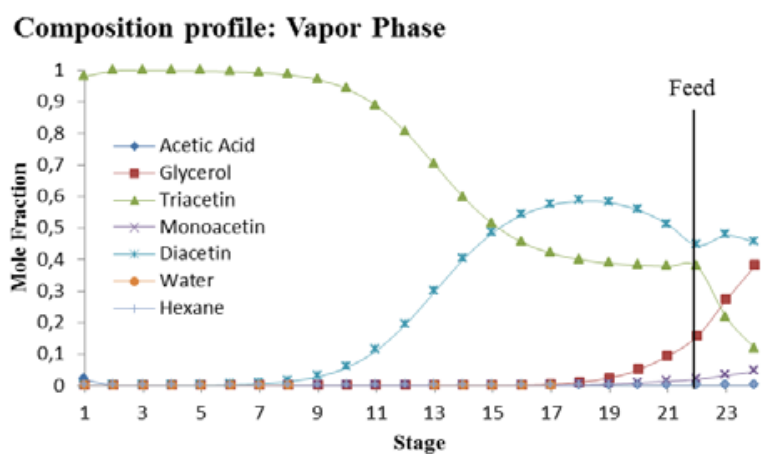

Fig. 15. Vapor liquid composition profile at column C3.

As a result, column C3 has 24 stages: the first stage is the condenser and the $24^{\text {th }}$ stage is the reboiler. The feed stream (stream 7 ) is fed at the $22^{\text {nd }}$ stage. The column pressure was set to $50 \mathrm{mmHg}(0.07 \mathrm{bar})$, and the reboiler operates at $201.6^{\circ} \mathrm{C}$ (Fig.13).

Fig. 14 and Fig. 15 show the molar fraction of the components in the liquid and vapor phases, respectively, in column C3. The top stream contains triacetin within the required specifications, while the bottom stream is rich in diacetin and glycerol. This stream is mixed with the bottom stream from C2 (stream 8, Fig. 3) and is returned to the reactive distillation column. The optimal reflux ratio in column C3 is 4, the condenser removal duty is $1478.4 \mathrm{~kW}$ and the reboiler duty $1478.5 \mathrm{~kW}$.

The main characteristics of the product obtained at the top of column C3 are shown in Table IV.

TABLE IV: PROCESS PRODUCT STREAM (STREAM 10)

\begin{tabular}{|c|c|c|}
\hline & Molar Flowrate $(\mathrm{kmol} / \mathrm{h})$ & Mole Fraction \\
\hline Acetic acid & 0.017 & 0.001 \\
\hline Glycerol & $1.8 \mathrm{E}-12$ & 10.9ppb \\
\hline Triacetin & 16.15 & 0.999 \\
\hline Monoacetin & $7.8 \mathrm{E}-11$ & 4.8ppb \\
\hline Diacetin & $2.03 E-3$ & 126ppm \\
\hline Water & 8.4E-6 & 0.52ppm \\
\hline Hexane & $2.5 \mathrm{E}-12$ & 15.7ppb \\
\hline Mass Flow (kg/hr) & \multicolumn{2}{|l|}{3523.5} \\
\hline Temperature $\left({ }^{\circ} \mathrm{C}\right)$ & \multicolumn{2}{|l|}{173.2} \\
\hline State & \multicolumn{2}{|l|}{ Liquid } \\
\hline
\end{tabular}

The hexane mass flowrate circulating in the reactive distillation column $\mathrm{C} 1$ is $126.7 \mathrm{t} / \mathrm{h}$. This flowrate is considerably larger than that for other entrainers, such as isobutyl acetate or ethylene dichloride. However, hexane is less expensive and requires less energy and smaller equipments to separate it from the other components.

The main characteristics of all streams represented in Fig. 3 are listed in Table V.

TABLE V: MAIN CHARACTERISTICS OF PROCESS STREAMS (OPTIMAL CONFIGURATION)

\begin{tabular}{|c|c|c|c|c|c|c|c|c|c|c|c|c|c|c|c|}
\hline & 1 & 2 & 3 & 4 & 5 & 6 & 7 & 8 & 9 & 10 & 11 & 12 & 13 & 14 & 15 \\
\hline \multicolumn{16}{|l|}{$\begin{array}{l}\text { Molar } \\
\text { Fraction }\end{array}$} \\
\hline Acetic acid & 0 & 0.95 & 0.049 & 0.021 & 0 & 0.69 & 0.0007 & 0 & 0.98 & 0.001 & 0.44 & 0.71 & 0.021 & trace & 0.002ppm \\
\hline Glycerol & 0.95 & 0 & 10.4ppm & 360ppb & 0 & 0.1 & 0.14 & 0.55 & $0.28 p p m$ & 10.9ppb & 1.6ppm & 95ppm & 360ppb & 0.58 & 0.47 \\
\hline Triacetin & 0 & 0 & $0.002 \mathrm{ppm}$ & 107ppb & 0 & 0.12 & 0.72 & 0.048 & 60ppm & 0.999 & 302ppm & $0.3 \mathrm{ppm}$ & 107ppb & 0.04 & 0.06 \\
\hline Monoacetin & 0 & 0 & $0.003 p p m$ & 10ppb & 0 & 0.012 & 0.017 & 0.063 & 926ppm & 4.8ppb & 403ppm & 663ppm & 10ppb & 0.066 & 0.06 \\
\hline Diacetin & 0 & 0 & $0.04 \mathrm{ppm}$ & 317ppb & 0 & 0.063 & 0.12 & 0.34 & 2.6ppm & 126ppm & 64ppm & 1.6ppm & 317ppb & 0.32 & 0.41 \\
\hline Water & 0.05 & 0.05 & 0.037 & 0.001 & 0 & 0.015 & $0.4 \mathrm{ppm}$ & 0 & 0.02 & 0.52ppm & 0.56 & 0.29 & 0.0012 & trace & trace \\
\hline Hexane & 0 & 0 & 0.91 & 0.977 & 1 & 0.0009 & $0.1 \mathrm{ppb}$ & 0 & 0.001 & 15.7ppb & 0.001 & 0.002 & 0.98 & trace & trace \\
\hline $\begin{array}{l}\text { Molar Flow } \\
(\mathrm{kmol} / \mathrm{h})\end{array}$ & 17 & 204 & 1606.8 & 1503.8 & 0.24 & 144.6 & 23.2 & 26.65 & 101.8 & 16.2 & 103.3 & 205.1 & 1503.6 & 19.6 & 7.0 \\
\hline $\begin{array}{l}\text { Mass Flow } \\
(\mathrm{kg} / \mathrm{h})\end{array}$ & 1502.6 & 11821.9 & 132400 & 128627 & 20.54 & 13002.1 & 4484.8 & 3450.2 & 6028.5 & 3523.5 & 3793.2 & 9821.6 & 3145 & 32.6 & 961.3 \\
\hline $\begin{array}{l}\text { Temperature } \\
\left({ }^{\circ} \mathrm{C}\right)\end{array}$ & 80 & 80 & 5 & 5 & 45 & 137.2 & 179.9 & 202.6 & 45.1 & 173.2 & 5 & 29.3 & 5 & 203 & 201.6 \\
\hline $\begin{array}{l}\text { Pressure } \\
\text { (bar) }\end{array}$ & 1 & 1 & 1 & 1 & 1 & 1 & 0.07 & 1 & 0.07 & 0.07 & 1 & 1 & 1 & 0.07 & 0.07 \\
\hline State & Liquid & Liquid & Liquid & Liquid & Liquid & Liquid & Liquid & Liquid & Liquid & Liquid & Liquid & Liquid & Liquid & Liquid & Liquid \\
\hline
\end{tabular}

\section{CONCLUSIONS}

In this paper, the design of an industrial triacetin production unit from glycerol was investigated, as a way to increase the feasibility of biodiesel production. First, the kinetic parameters obtained by Galan et al. [2] were analyzed and applied to a reactive distillation column. By means of planned simulations, the structure of the reaction and separation units was defined, and the specifications for the processing units and process streams were selected. The operational conditions of the columns were studied in order to accomplish the product requirements and minimize the energy consumption. The proposed configuration is able to completely convert glycerol to triacetin with high level of purity (99.9 \%). The amount of heat removed from the condensers of the entire process corresponds to $21.9 \mathrm{MJ} / \mathrm{kg}$ of product, and the amount of heat required at the reboilers is 


\subsection{MJ/kg of product.}

The information provided in this paper can be adopted as a basis for specific feasibility studies at a conceptual process design level.

\section{REFERENCES}

[1] N. Bremus, G. Dieckelmann, L. Jeromin, W. Rupilius, H. Schutt, and H. K. auf Aktien, "Process for the continuous production of triacetin," U.S. Patent 4381407, 1981.

[2] M.-I. Galan, J. Bonet, R. Sire, J.-M. Reneuaume, and A. E. Plesu, "From residual to useful oil: Revalorization of glycerin from the biodiesel synthesis,” Bioresource Technology, vol. 100, pp. 3775-3778, 2009.

[3] S. K. Hung, C. C. Lee, H. Y. Lee, C. L. Lee, and I. L. Chien, "Improved design and control of triacetin reactive distillation process for the utilization of glycerol," Industrial \& Engineering Chemistry Research, vol. 53, pp. 11989-12002, 2014.

[4] C. V. Lacerda, M. J. Carvalho, A. R. Ratton, I. P. Soares, and L. E. Borges, "Synthesis of triacetin and evaluation on motor," J. Braz. Chem. Soc., vol. 26, no. 8, pp. 1625-1631, 2015.

[5] X. Liao, Y. Zhu, S.-G. Wang, and Y. Li, "Producing triacetylglycerol with glycerol by two steps: Esterification and acetylation," Fuel Processing Technology, vol. 90, pp. 988-993, 2009.

[6] V. L. Gonçalves, B. P. Pinto, J. C. Silva, and C. J. Mota, "Acetylation of glycerol catalyzed by different solid acids," Science Direct Catalysis Today, vol. 133-135, pp. 673-677, 2008.

[7] Z. Mufrodi, S. Rochmadi, and A. Budiman, "Chemical kinetics for synthesis of triacetin from biodiesel byproduct," International Journal of Chemistry, vol. 4, pp. 101-107, 2012.

[8] Ministério de Minas e Energia Brasileiro, "Boletim Mensal dos Biocombustíveis," Monthly Bulletin of Biofuels, Edition n¹06, Dec. 2016.
[9] X. Liao, Y. Zhu, S.-G. Wang, H. Chen, and Y. Li, "Theoretical elucidation of acetylating glycerol with acetic acid and acetic anhydride," Appl. Catal. B., vol. 94, pp. 64-70, 2010.

[10] P. Atkins and J. Paula, Atkins'Physical Chemistry, New York, Oxford University Press, 2006, pp. 200-234.

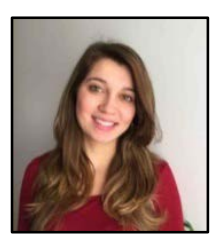

Tatiane Fernandes Caetano Souza earned her chemical engineering degree in 2013 from Escola Politécnica da Universidade de São Paulo (EPUSP), Brazil, and her industrial/generalist engineering degree in 2014 from Ecole Centrale de Lille, France. Presently she is pursuing a master degree in the field of chemical engineering with emphasys on process engineering design and simulation in the Chemical Engineering Department at Escola Politécnica da Universidade de São Paulo (EPUSP). She has 4 years' experience on working as a process engineer for a project engineering company from Brazil, Promon Engenharia.

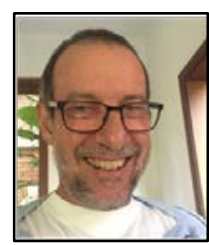

Roberto Guardani is chemical engineer, professor at the Chemical Engineering Department, University of São Paulo, carries out research in the area of process systems engineering, involving: Mathematical modeling, simulation and optimization; multivariate statistical modeling, applied mainly in the following subjects: Industrial effluents treatment; atmospheric emissions; processes involving particles, with emphasis on fluidization, crystallization, multiphase flow; development of in-process measurement techniques. 